\title{
Aprovisionamento Automático de Circuitos Ópticos Protegidos via Plano de Controle GMPLS Considerando Restrições de Camada Física
}

\author{
G.C. Santos, F.D. Santos, J.C.R.F. Oliveira, R.A. Moreira, M.R. Salvador, M.A. Romero e M. Magalhães
}

\begin{abstract}
Resumo - O aprovisionamento automático de circuitos ópticos protegidos via Plano de Controle GMPLS considerando restrições de camada física (atenuação e PMD) é demonstrado experimentalmente em um cenário de uma rede óptica reconfigurável baseada em ROADMs de grau 2. Foram alteradas as mensagens de disseminação de informações topológicas e de sinalização, permitindo a construção de um algoritmo de RWA capaz de usar estas informações para calcular rotas que interfiram minimamente na natureza do sinal transmitido. Foi proposta uma integração entre Planos de Controle e de Dados para deteç̧ão e recuperação de falhas nos enlaces de maneira rápida e eficiente.

Palavras-Chave - GMPLS, RWA, Proteção.
\end{abstract}

\begin{abstract}
The provisioning of protected optical circuits using GMPLS Control Plane and considering physical impairments (link attenuation and PMD) is experimentally demonstrated using a reconfigurable optical network based on 2-degree ROADMs. To achieve this goal, messages related to signaling and topological information flooding were changed. Then, an RWA algorithm could be defined and implemented, which is able to use these informations to calculate paths which interfere minimally on the transmitted signal nature. Also, it is proposed a integration between Data and Control Planes to detect and recovery link failures quickly and efficiently.
\end{abstract}

Keyworks - GMPLS, RWA, Protection.

\section{I.INTRODUÇÃO}

A estrutura atual das redes de telecomunicações apresenta uma capacidade de transmissão e recepção de dados que em poucos anos não será mais capaz de atender toda a demanda existente. Com isso, novos sistemas de comunicação têm surgido, baseados em uma infraestrutura óptica interligando backbones e redes metropolitanas. Consequentemente, cresce também a necessidade de implementar mecanismos seguros e eficientes para o controle e o gerenciamento dessas novas redes. Entre as tarefas requeridas, estão a realização automática do aprovisionamento e a proteção e restauração de rotas entre elementos da rede que utilizem estas fibras ópticas.

$\mathrm{Na}$ atual conjuntura do desenvolvimento das redes ópticas, é cada vez mais frequente a presença de elementos reconfiguráveis na topologia. Estes elementos são capazes de

G.C. Santos, F.D. Santos, J.C.R.F. Oliveira, R.A. Moreira, M.R.Salvador, Diretoria de Redes de Telecomunicações; Fundação CPqD, Campinas, Brasil. Emails: \{gcuriel; fabios; julioc; rmoreira; marcosrs\}@cpqd.com.br.

M.A. Romero, Escola de Engenharia, USP, São Carlos, Brasil. muriloa@sel.eesc.usp.br.

M. Magalhães, Faculdade de Engenharia Elétrica e Computação, Unicamp, Campinas, Brasil.mauricio@dca.fee.unicamp.br realizar a inserção e remoção de canais em determinados pontos da rede, permitindo a reconfiguração desta em um curto intervalo de tempo. A presença deste tipo de equipamento é fundamental para que processos automáticos para criação de caminhos sejam usados [1].

Idealmente, o operador da rede WDM (Wavelength Division Multiplexing) necessitaria apenas fornecer a origem e destino da rota (e possivelmente informações adicionais de Engenharia de Tráfego). A partir daí, protocolos capazes de mapear a topologia física em uma representação lógica fornecem dados para que algoritmos especializados, conhecidos como RWA (Routing and Wavelength Assignment), calculem a melhor rota entre estes dois pontos e escolham um comprimento de onda adequado. Além disso, é importante que o sinal transmitido tenha suas propriedades ópticas minimamente alteradas ao atravessar a rota calculada. Assim, estes algoritmos devem levar em consideração, além da topologia e comprimentos de onda disponíveis, restrições físicas relacionadas ao meio de transmissão. Após o cálculo de rota, mensagens de sinalização são trocadas entre os elementos para reservar os recursos necessários para a implantação do caminho.

Para aumentar a confiabilidade das rotas existentes na rede, uma das maneiras mais utilizadas pelos provedores de serviços é assegurar redundância de conexões, as quais se baseiam na replicação de recursos na rede (transmissores, comutadores ópticos, amplificadores). Para garantir o bom uso destes recursos replicados, deve existir a possibilidade de cálculo e alocação de caminhos diversos entre dois nós. Estes podem ser usados tanto como caminhos principais como caminhos de reserva. Assim, ficam previstas configurações para que, caso ocorra alguma falha, um elemento de rede possa detectá-la e localizá-la tão rapidamente quanto possível, podendo comutar o caminho usado para a transmissão de dados.

No contexto acima, observando as considerações citadas, são apresentadas neste artigo as customizações feitas em um Plano de Controle GMPLS (Generalized Multiprotocol Label Switching) visando oferecer ferramentas para a criação de caminhos ópticos considerando restrições da camada física e um mecanismo de proteção baseado em uma solução composta por elementos de hardware e software.

Este trabalho está dividido na seguinte forma: a seção II apresenta os conceitos do GMPLS. Nas seções III e IV são apresentadas as considerações feitas neste trabalho sobre algoritmos de RWA e proteção de redes, respectivamente, 
seguido dos resultados, mostrados na seção $\mathrm{V}$.

\section{II.GMPLS}

Um grande número de redes de dados atualmente instaladas baseia-se no processo de comutação de pacotes, empregando em seus elementos de roteamento tabelas baseadas em endereços IP. O encaminhamento de um pacote IP é feito através da busca do endereço da rede de destino, presente na tabela de roteamento, que mais se aproxima do endereço de destino contido no pacote. Desta forma, o pacote é transmitido pela interface que possui acesso a esta rede. Cada roteador deve fazer esta busca para a decisão do próximo nó ao qual o pacote será transmitido. Estes procedimentos são baseados em entrega por melhor esforço, não sendo possível ter garantia alguma de qualidade de serviço $(Q o S)$. Desta forma, foi criada uma tecnologia chamada MPLS (Multiprotocol Label Switching) [2], baseada no uso de rótulos nos pacotes. Quando os pacotes passam pelos roteadores de entrada da rede, são separados em classes e, para cada uma delas, é atribuído um rótulo. Em um roteador intermediário, este rótulo (juntamente com a interface por onde o dado foi recebido) é associado a outro par rótulo/interface de saída. Desta forma, é possível a criação de circuitos virtuais, admitindo-se também a separação dos pacotes em classes, o que possibilita a garantia dos requisitos de QoS definidos.

O GMPLS é uma evolução que agrega ao MPLS a capacidade de considerar não apenas rótulos para comutação de dados, mas também informações sobre os meios de transmissão de dados - como comprimentos de onda e fibras -, ou sobre o modo como os dados são transmitidos (como pacotes ou janelas temporais). Assim, a rede passa a ser representada como uma combinação de camadas lógicas, cada uma responsável por trabalhar com uma determinada tecnologia de transporte (pacotes, WDM, comutação de fibras). A integração destas camadas oferece uma visão unificada da topologia [3].

$\mathrm{Na}$ arquitetura GMPLS, a rede é dividida em planos independentes: Plano de Dados, de Controle e de Gerência. O Plano de Dados é composto pela estrutura que irá transmitir efetivamente os dados (comutadores, amplificadores, entre outros), enquanto que no Plano de Controle são trocadas mensagens a respeito do Plano de Dados e sobre as decisões de quais configurações deverão ser feitas nos equipamentos deste plano. Já o Plano de Gerência é voltado para os operadores e administradores da rede, e se preocupa em apresentar informações estatísticas da rede, além de atuar como canal de entrada e saída de informações de controle da rede. É por ele que são feitos os pedidos de criação e remoção de rotas, ou o cadastro de novas informações de Engenharia de Tráfego no sistema [4].

\section{III.RWA}

Para que uma conexão em uma rede óptica seja feita entre dois pontos, é necessário que seja definido, além da rota, um comprimento de onda. Este problema, conhecido como RWA, pode ser formulado como um programa linear inteiro misto, já que é NP-completo [5]. Este problema se torna mais tratável se for particionado em dois subproblemas: (a) roteamento e (b) atribuição de comprimentos de onda. Cada um destes subproblemas pode ser resolvido separadamente. Para o subproblema de roteamento, há três abordagens básicas encontradas na literatura: roteamento fixo, roteamento fixoalternativo e roteamento adaptativo [6]. Para a atribuição do comprimento de onda, algumas heurísticas foram propostas, tais como: atribuição randômica de comprimento de onda, First-Fit, Least-Used, Most-Used, Min-Product, Least Loaded, MAX-SUM, Relative Capacity Loss, Wavelength Reservation e Protecting Threshold ([7], [8], [9], [10], [11], [12], [13]).

A construção de um RWA para uso real deve levar em conta não só a disponibilidade de um comprimento de onda, mas também algumas particularidades de implementação e operação das redes ópticas. Estas considerações devem ser relacionadas à existência de certas classes de equipamentos na rede e suas limitações de operação, além de parâmetros físicos inerentes aos equipamentos de transmissão.

Uma das considerações que devem compor um RWA eficiente é a existência ou não de conversores de comprimento de onda na topologia. No cenário atual das redes de telecomunicações, é tipicamente necessário que o mesmo comprimento de onda seja utilizado em todos os enlaces para transmissão do sinal óptico, problema conhecido como limitação de continuidade de comprimento de onda. Além disso, o sinal transmitido sofre várias degradações enquanto atravessa a rede, causadas por imperfeições inerentes aos meios de transmissão e pelo funcionamento não-ideal dos equipamentos intermediários. Quando se considera uma rede sem regeneração de sinal, a inclusão destes fatores no cálculo de rota se torna muito importante já que, sem ela, pode-se ter um caminho criado com tantas degradações que o tornem inutilizável.

Outra peculiaridade das redes ópticas é a alta assimetria de alguns de seus elementos, não sendo possível a conexão de qualquer porta de entrada a qualquer porta de saída. Por exemplo, a estrutura interna de um ROADM PLC (Reconfigurable Optical Add/Drop Multiplexer - Planar Lightwave Circuit) de 40 portas é mostrada na Figura 1.

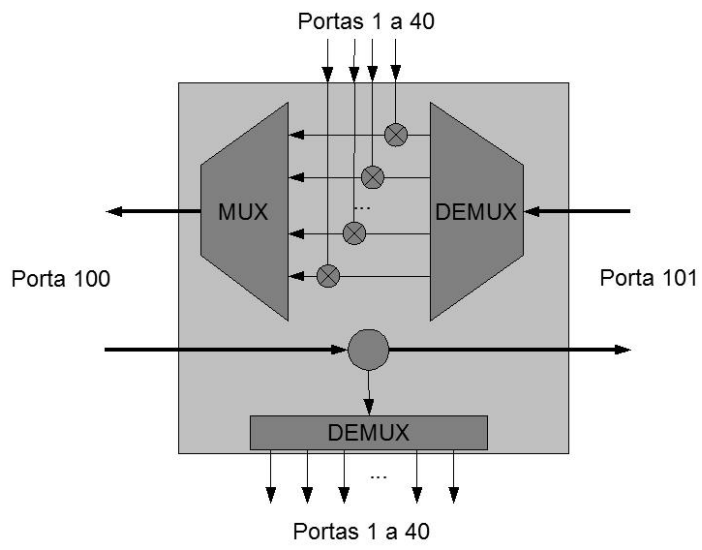

Figura 1: Estrutura interna de um ROADM PLC de Grau 2. 
Pela figura, por construção, as portas de 1 a 40 não podem ser ligadas à porta 101, mas apenas à porta 100. Desta forma, os caminhos que não levam em consideração esta característica do equipamento podem não ser utilizáveis, já que exigiria uma configuração impossível do equipamento do Plano de Dados.

Neste trabalho foi construído um algoritmo de RWA que leva em consideração os seguintes fatores, além da disponibilidade de comprimento de onda:

-Restrições físicas: PMD (Polarization Mode Dispersion) e atenuação;

-Restrições de funcionamento dos equipamentos devido à assimetria dos mesmos.

Este algoritmo foi implementado para ser usado em redes em malha de forma online, o que obriga a obtenção destas informações de forma dinâmica, através de mensagens do protocolo OSPF-TE (Open Shortest Path First - Traffic Engineering) [14]. A solução para isto foi a alteração dos LSAs (Link State Advertisements) para que, além das informações de enlace trocadas entre as instâncias do protocolo, fossem disseminadas outras estruturas, contendo as restrições já citadas, que podem ser usadas pelo algoritmo de RWA. As estruturas adicionadas foram:

- Sub-TLV Lista de comprimentos de onda: possui uma lista de comprimentos de onda que a interface consegue enviar e receber. Ela é usada quando da seleção do comprimento de onda pelo RWA.

- Sub-TLV Restrições físicas: possui uma lista fixa de restrições físicas (PMD e atenuação), as quais são representadas cada uma por um valor de ponto flutuante de quatro bytes. Estes valores foram lidos a partir de arquivos de configuração;

- Sub-TLV Grupo de conexão: possui duas listas de grupos de conexão, uma para conexões de entrada e outra para conexões de saída. Se duas ou mais interfaces pertencem ao mesmo grupo de conexão em listas diferentes significa que elas podem ser ligadas pelo equipamento. Esta informação tem significado local, permitindo que dois nós possuam o mesmo grupo. Um exemplo é apresentado na Figura 2, onde são mostradas as portas do ROADM com seus respectivos grupos de conexão de entrada e saída (Grupo: ENTRADA/SAÍDA). Desta forma, as portas de acesso podem ser conectadas à porta 100 , já que estão no grupo 1 , e as portas 100 e 101 podem ser conectadas, uma vez que estão no grupo 2 .

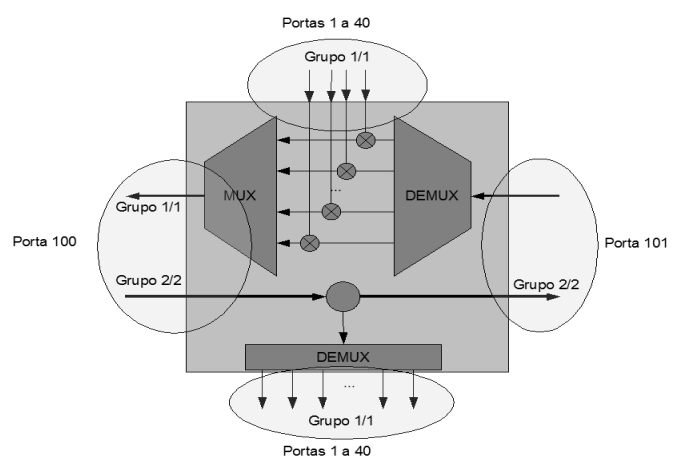

Figura 2: ROADM com grupos de conexão
Com estas alterações feitas no OSPF-TE, foi definido e implementado o algoritmo de RWA usando conceitos de algoritmos genéticos. As estruturas básicas do algoritmo são:

-Genes: pares enlace/nó conectados entre si;

-Indivíduos: conjuntos de genes (nós e enlaces) conectados de forma que possam formar uma rota;

-População: conjunto de indivíduos (caminhos), distintos entre si ou não, que têm origem e destino iguais aos da requisição de cálculo de rota. Não é obrigatório que estes indivíduos possuam um comprimento de onda ou respeitem as restrições de conexão dos equipamentos.

O funcionamento geral do algoritmo é mostrado na Figura 3. A função-aptidão (fitness) é calculada a partir da soma de todas as restrições físicas, da existência ou não de um comprimento de onda disponível em todas as interfaces da rota, da possibilidade de configuração da rota devido a assimetria dos equipamentos e do número de saltos do caminho. Assim, quanto menor o fitness de uma rota, menos propícia para escolha ela fica. Caso exista alguma configuração que não permita a existência física do indivíduo (como, por exemplo, a falta de um comprimento de onda), é atribuído a ele um fitness muito baixo, porém este indivíduo não é eliminado da população. Para se ter um maior controle sobre o peso que uma restrição tem sobre o resultado final, todas as restrições físicas são normalizadas.

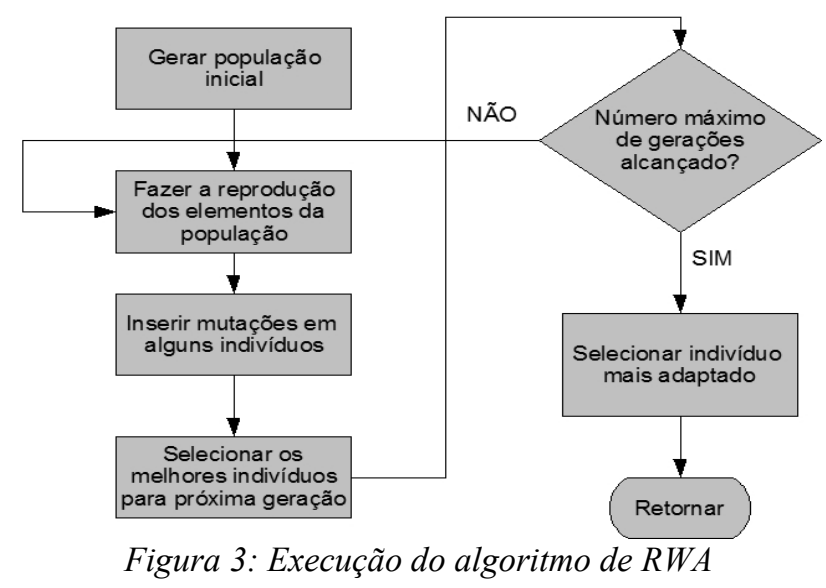

A geração da nova população é feita através de torneio binário, onde dois elementos são selecionados aleatoriamente para reprodução. Embora este procedimento dificulte a convergência do algoritmo, ele torna menos provável a existência de super indivíduos, os quais provocariam a convergência prematura para um valor ótimo local. Além disso, existe um controle de indivíduos gêmeos, para aumentar a variabilidade genética da população. As mutações inseridas em alguns indivíduos são realizadas através da eliminação de parte da rota e posterior recálculo, através do algoritmo de Dijkstra, da parte eliminada.

Quando o número máximo de gerações de população é atingido, o indivíduo mais adaptado é escolhido, e é feita nele 
uma busca por inconsistências. Nesta busca, é verificado se há ao menos um comprimento de onda disponível em toda a rota e se serão necessárias apenas configurações válidas dos equipamentos, respeitando as limitações de operação dos mesmos. Se algum destes dois testes falhar, conclui-se que não há rota que atenda a requisição, e um erro é retornado indicando esta situação. Caso contrário, a rota é encaminhada para o protocolo de sinalização.

Para a obtenção dos resultados deste trabalho, foi usada a topologia mostrada na Figura 4. Por simplicidade do cenário de testes, alguns elementos do plano de dados foram retirados, embora os enlaces do plano de controle aqui sejam usados como representação do plano físico.

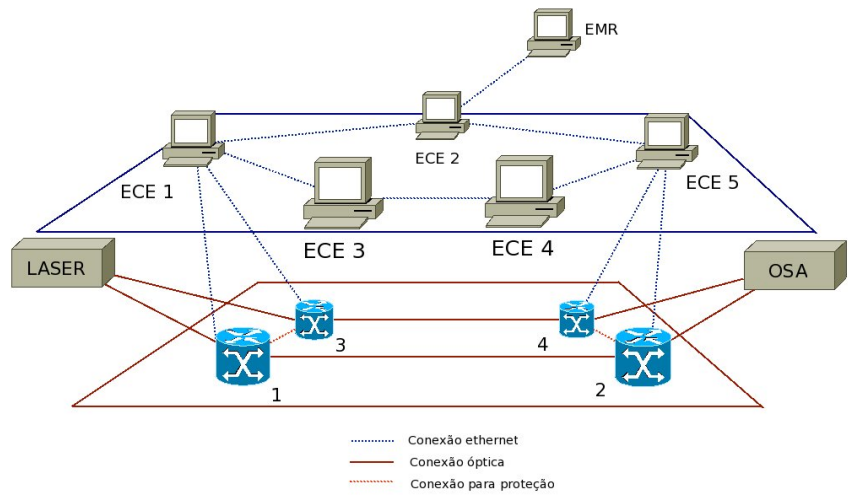

Figura 4: Topologia lógica utilizada.

Nesta topologia, os Elementos de Controle de Equipamentos (ECEs) são os representantes lógicos no Plano de Controle dos equipamentos físicos do Plano de Dados, disseminando informações sobre os mesmos e enviando configurações a eles. Já o Elemento de Manipulação de Requisições (EMR) é responsável pelo cálculo de rota e controle dos caminhos criados na rede.

Os enlaces desta topologia possuem as características enumeradas na Tabela 1. A influência da PMD é analisada através do valor da DGD média (Differential Group Delay).

\begin{tabular}{|c|c|c|c|}
\hline Enlace & Canal & DGD média (ps) & Atenuação (dB) \\
\hline $1-2$ & 27 & 0.2 & 1.4 \\
\hline $2-5$ & 27 & 0.2 & 1 \\
\hline $1-3$ & $27-29$ & 0.2 & 1.83 \\
\hline $3-4$ & $27-29$ & 0.2 & 2 \\
\hline $4-5$ & $27-29$ & 0.2 & 1.5 \\
\hline
\end{tabular}

Tabela 1: Relação entre enlaces, PMD e atenuação nos canais da topologia

\section{IV.Proteção eM REDES GMPLS}

Todas as redes de comunicação estão sujeitas a falhas, as quais podem impedir sua operação e funcionamento. Em redes ópticas, estas falhas podem ocorrer em enlaces, elementos de comutação ou mesmo nos canais de controle.

A detecção da falha é o primeiro requisito para que seja possível oferecer proteção para as conexões. A operacionalidade de um enlace pode ser inferida através do acompanhamento de parâmetros específicos dos equipamentos, como alarmes gerados pelos comutadores ópticos ou amplificadores. Outra possibilidade consiste no uso de protocolos construídos para tal função, como o LMP (Link Management Protocol) [15].

Assim que uma falha é detectada, é necessário localizar em qual ponto exatamente o problema aconteceu. Em redes ópticas, é comum que um problema detectado em um caminho seja identificado por todos os equipamentos que o compõem. Por isso, deve existir algum modo de interação entre os Planos de Dados e de Controle para obter o ponto exato do problema. Encontrado o local da falha, esta é informada ao elemento que gerencia os recursos da rede através do protocolo de sinalização. Esta inteligência é responsável por comunicar o evento a um nível mais alto de gerência - normalmente o operador da rede -, e iniciar o processo de restauração de acordo com as políticas definidas para cada tipo de falha que pode ocorrer no sistema.

Existem várias abordagens para o processo de restauração do sistema [16]. Entre elas, duas se destacam como sendo as mais estudadas e utilizadas. A primeira atua localmente, isolando o trecho ou elemento onde ocorreu o problema, realizando um redirecionamento do tráfego para outro nó. Este abordagem realiza os cálculos e configurações necessárias após a falha ter acontecido.

A segunda abordagem leva em conta a capacidade dos elementos de início e fim de rota de comutar entre caminhos de trabalho e de proteção, a partir da notificação de uma falha. Esta abordagem é chamada de proteção fim-a-fim. Estes caminhos de proteção são previamente calculados (e em alguns casos casos, reservados) pelo sistema antes que uma falha aconteça [17].

Considerando as definições encontradas na literatura, observa-se que as ações de proteção e restauração das redes GMPLS são concentradas no Plano de Controle, que considera os equipamentos do Plano de Dados como elementos passivos destas ações. Esta característica implica que o sistema pode não ser rápido o bastante para evitar que o serviço seja interrompido caso uma falha aconteça em uma rede óptica [18]. Assim, levando em conta as particularidades das redes ópticas no que diz respeito ao processo de detecção e restauração de falhas [19], esquemas de proteção estudados e referenciados e tipos de abordagens para a restauração do sistema, foi proposto um mecanismo de proteção para redes ópticas baseadas em ROADMs de grau 2.

Este mecanismo utiliza o esquema de proteção fim-a-fim dedicada 1:1 sem tráfego extra, no qual um caminho de proteção é calculado e pré-alocado para cada caminho primário, sem que haja replicação do sinal. A detecção e recuperação da falha é feita diretamente no Plano de Dados, pelos ROADMs. Assim que um problema é detectado pelo equipamento (por exemplo, LOS - Loss of Signal), automaticamente o sinal é comutado para o caminho de proteção, caso esteja configurado para realizar tal ação. Neste caso, o papel do Plano de Controle é de calcular e reservar um caminho de proteção na topologia, e configurar os 
equipamentos no Plano de Dados para que a ação de proteção possa ser realizada. É importante observar que este chaveamento é feito por comprimento de onda, ou seja, podem haver vários caminhos que utilizam a mesma fibra, porém apenas aqueles que tiverem um caminho de proteção configurados terão seus sinais redirecionados.

O processo de cálculo do caminho de proteção é feito da mesma maneira que a descrita no processo de cálculo de rotas (seção III), com uma exceção importante. Para o caminho de trabalho, todos os enlaces da topologia são considerados, visando encontrar o melhor caminho entre os dois pontos de entrada e saída da rede. Tão logo este caminho seja computado, os enlaces que o compõem são desconsiderados para o cálculo da rota de proteção. Esta atitude garante que, caso exista um caminho de proteção, este será completamente disjunto do caminho de trabalho. Na prática, essa ação resulta em uma maior garantia de que uma falha no enlace não atinja ambos os caminhos simultaneamente.

Algumas características particulares das redes ópticas foram observadas. Entre elas, a possibilidade de escolher manualmente um comprimento de onda específico para um determinado caminho calculado pelo Plano de Controle. $\mathrm{Na}$ prática, um comutador óptico pode não conter fibras em todas as suas portas e, consequentemente, deve-se evitar que o Plano de Controle (que possui a capacidade de escolher comprimentos de onda automaticamente) escolha um caminho com um comprimento de onda não disponível.

Outro ponto importante é a escolha do mesmo comprimento de onda tanto para o caminho de trabalho quanto o de proteção. A topologia mostrada na Figura 4, a qual serviu de base para este trabalho, impõe que o mesmo canal do caminho principal seja usado para o caminho de proteção para que o mecanismo de recuperação de falhas funcione de maneira correta e eficiente.

A indicação de que a rota deverá ser calculada com proteção (ou não) é feita em um bit do campo de opções da requisição. Já na sinalização, foram usados identificadores presentes nas mensagens de sinalização para distinguir as rotas de trabalho e de proteção. Estas informações são necessárias para a configuração dos equipamentos no Plano de Dados.

\section{V.RESULTADOS}

Para os testes deste esquema de proteção foi usada a topologia mostrada na Figura 4. As máquinas ECE 1 e ECE 5 são máquinas reais com duas interfaces de rede, uma para comunicação com os outros elementos do Plano de Controle e outra para comunicação com os ROADMs. Já os outros ECEs (ECE 2, ECE 3 e ECE 4) são instâncias virtuais iniciadas no UML (User Mode Linux), e seus enlaces são implementados através de túneis GRE. A conexão de proteção entre os ROADMs é necessária para comunicação direta entre estes elementos no caso de falha do enlace, dando início ao procedimento de troca do enlace usado para comunicação. $\mathrm{O}$ quadro a seguir mostra a saída do sistema para o cálculo de uma rota protegida entre ECE 1 e ECE 5.
08:05:21: Tratamento da mensagem com parametro de protecao 08:05:21: Verificando requisicao

08:05:21: Calculando rota principal, sem forcar lambda

08:05:22: Primeira Populacao:

08:05:22: No. de individuos: 250

08:05:22: Primeiros Individuos:

08:05:22: (0) (0, \#०) Hops: 125

08:05:22: (0) $(0, \# 0)$ Hops: 125

08: $05: 22:(0)(0, \# 0)$ Hops: 125

08:05:22: (0) (0, \#०) Hops: 125

08:05:22: Populacao final :

08:05:22: No. de individuos: 1

08: 05:22: Individuos:

08:05:22: (1.04812, \#1 27) Hops: 125

08:05:22: Alocando canal: 27

08:05:22: Inserindo roteadores na lista do LSP.

08:05:22: Canal escolhido: 27, Atenuacao: 2, PMD: 0.83, Fitness: 1.04812

08:05:22: Bloqueando links

08:05:22: LINK@10.5.1.1[12.1.2.1-12.1.2.2]

08:05:22: LINK@10.5.1.2[12.2.5.1-12.2.5.2]

08:05:22: Calculando rota de protecao...

08:05:22: Forcando comprimento de onda do RWA para 27

08:05:22: Primeira Populacao:

08:05:22: No. de individuos: 250

08: 05:22: Primeiros Individuos:

08:05:22: (0) $(0, \# 0)$ Hops: 1345

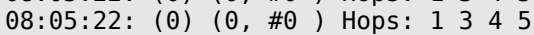

08:05:22: (0) (0, \#0) Hops: 1345

08:05:22: (Ө) (0, \#०) Hops: 1345

08:05:22: Populacao final:

08:05:22: No. de individuos: 28

08:05:22: Primeiros Individuos:

08:05:22: (1.44276, \#3 272829$)$ Hops: 1345

08:05:22: (1.44276, \#3 272829$)$ Hops: 1345

08:05:22: (1.44276, \#3 272829$)$ Hops: 1345

08:05:22: (1.44276, \#3 272829 ) Hops: 1345

08: $05: 22$ : Alocando canal: 27

08:05:22: Inserindo roteadores na lista do LSP:

08:05:22: Canal escolhido: 27, Atenuacao: 7.43, PMD: 0.6 , Fitness: 1.44276

As rotas calculadas são apresentadas juntamente com o fitness na forma (fitness, \#N L) Hops: H, onde N é o número de comprimentos de onda disponíveis para aquela rota, L são os canais disponíveis, e H é uma lista com identificadores dos nós participantes da rota.

Ao receber as rotas, o ECE 1 inicia o processo de sinalização e alocação dos recursos. No processo de sinalização, cada rota gera uma mensagem de criação de caminho (mensagem PATH [20]) que é enviada através dos elementos que compõem a mesma até chegarem ao destino. $\mathrm{O}$ envio das duas mensagens é mostrado a seguir.

08:05:22.647 **** Nova Mensagem Recebida ****

08:05:22.650 Recebida mensagem PATH para

$10.5 .1 .5 / 2001,16844042$

$08: 05: 22.650$ Nova Sessao: 10.5.1.5/2001/16844042

08:05:22.674 Buscando endereco da interface de dados..

08:05:22.680 Rota encontrada do 0SPF:

08: $05: 22.680$ ECE: inPort: 0

$08: 05: 22.680$ ECE: outPort: 100

08:05:22.680 ECE: switchIDUpstream: 0.0 .0 .0

08: $05: 22.680$ ECE: switchIDDownst ream: 10.5.1.100

$08: 05: 22.681$ ECE: Tentando obter vendedor do equip..

08:05:22.689 ECE: Equipamento reconhecido: ROADM

08:05:22.689 ECE: Vendedor reconhecido, CPqD

08: 05:22.699 ECE: Criando controlador para o ROADM...

08:05:22.700 Inicializando controlador do R0ADM

08:05:22.701 ECE: SNMP estah habilitado

08:05:22.788 **** Nova Mensagem Recebida ****

08:05:22.798 Recebida mensagem PATH para

$10.5 \cdot 1.5 / 2002,16844042$

$08: 05: 22.798$ Nova Sessao: 10.5.1.5/2002/16844042

08:05:22.850 Buscando endereco da interface de dados..

08:05:22.852 Rota encontrada do OSPF: 


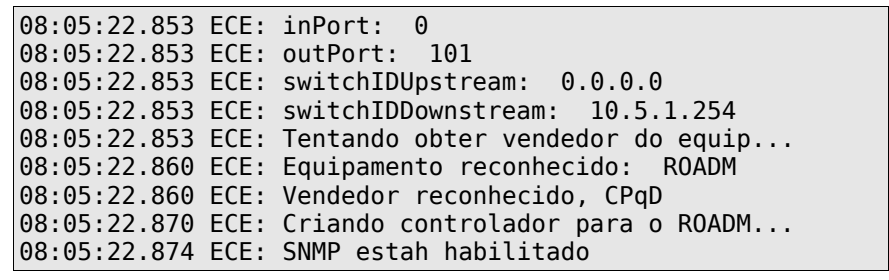

Quando as mensagens PATH são recebidas, o nó destino envia uma mensagem de reserva de caminho (mensagem RESV [20]) para reservar os recursos e confirmar a criação da rota. Assim, no elemento de origem, surgem dois caminhos criados, sendo que um deles é o de proteção do outro. No Plano de Dados, o que diferencia um caminho ativo de trabalho para um caminho de proteção é a configuração da porta de entrada e saída do sinal óptico no equipamento de comutação. Através de uma flag, é possível indicar se a porta utilizada para uma determinada rota é de trabalho ou de proteção. A partir disso, o próprio equipamento dispõe de mecanismos de deteç̧ão de erros que o permitem comutar o sinal entre o caminhos reservados previamente.

Assim, após a sinalização dos caminhos calculados no Plano de Controle, os equipamentos terão dois tipos de portas configuradas. As do caminho principal serão mostradas como portas de trabalho ativas, enquanto as que compõem a rota de proteção serão indicadas como portas de proteção inativas. Caso haja a necessidade de comutação de caminhos, as portas passam a indicar porta de trabalho inativa e porta de proteção ativa, respectivamente. A configuração no primeiro nó para o caminho de trabalho pode ser vista através da saída do sistema mostrada a seguir.

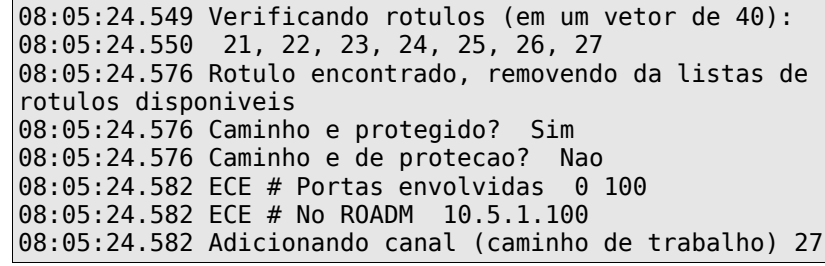

Para o caminho de proteção as configurações feitas, também no primeiro nó, são as seguintes:

08: $05: 25.463$ Verificando rotulos (em um vetor de 40 ) :
$08: 05: 25.463$ 21, 22, 23, 24, 25, 26, 27
08: $05: 25.463$ Rotulo encontrado, removendo da listas de
rotulos disponiveis
$08: 05: 25.463$ Caminho e protegido? Sim
$08: 05: 25.463$ Caminho e de protecao? Sim
$08: 05: 25.467$ ECE \# Portas envolvidas $\odot 101$
$08: 05: 25.467$ ECE \# No R0ADM 10.5.1.254
$08: 05: 25.467$ Adicionando canal (caminho de protecao) 27

É importante notar que em todas as configurações são verificadas os parâmetros de proteção, que definirão os comandos a serem enviados ao equipamento.

\section{VI.CONCLUSÃo}

$\mathrm{O}$ aprovisionamento de circuitos ópticos protegidos considerando restrições de camada física foi feito de forma automática, usando para isto adaptações no plano de controle. Foi criado, para o completo funcionamento do controle da rede um algoritmo de RWA considerando atenuação e PMD e um procedimento para cálculo e alocação de rotas de proteção, de onde surgiu a necessidade de alteração dos protocolos constituintes do GMPLS. Desta forma, abre-se espaço para a consideração de restrições físicas mais complexas, já que os elementos que deverão sofrer alteração para que o algoritmo receba os dados referentes a estas restrições já foram identificados. Além disso, também foi criada a base para a criação de outros procedimentos de proteção, integrando soluções do Plano de Controle com características dos equipamentos que compõem a rede.

\section{REFERÊNCIAS}

[1] ELADA, L. et. al. 40-channel ultra-low-power compact PLC-based ROADM subsystem OFC 2006, Anaheim, Estados Unidos, 2006.

[2] Rosen, E., Viswanathan, A., Callon, R. Multiprotocol Label Switching Architecture RFC 3031 Janeiro de 2001.

[3] Santos, G.C., Santos, F.D., Oliveira, J.C.R.F. Aprovisionamento Automático de Circuitos Ópticos via Plano de Controle GMPLS Aplicado a uma Rede Óptica Reconfigurável Baseada em ROADMs, SbrT 2008, Rio de Janeiro, Brasil.

[4] Mannie, E. Generalized Multi-Protocol Label Switching (GMPLS) Architecture RFC 3945, Outubro de 2004.

[5] I. Chlamtac, A. Ganz, and G. Karmi. Lightpath communications: an approach to highbandwidth optical WAN's. IEEE Transactions on Communications, 40(7):1171-1182, Julho 1992.

[6] Zang, H., Jue, J. P., Biswanath M. (2000) A Review of Routing and Wavelength Assignment Approaches for Wavelength-Routed Optical WDM Networks.

[7] I. Chlamtac, A. Ganz, and G. Karmi, "Purely Optical Networks for Terabit Communication," Proc., IEEE INFOCOM.

[8] R. A. Barry and S. Subramaniam. "The MAX-SUM Wavelength Assignment Algorithm for WDM Ring Networks," Proc., OFC '97, Feb. 1997.

[9] A. Birman and A. Kershenbaum, "Routing and Wavelength Assignment Methods in Single-Hop All- Optical Networks with Blocking," Proc., IEEE INFOCOM '95, Boston, MA, vol. 2, pp. 431-438, April 1995.

[10] G. Jeong and E. Ayanoglu, "Comparison of Wavelength- Interchanging and Wavelength-Selective Cross-Connects in Multiwavelength All-Optical Networks," Proc., IEEE INFOCOM '96, San Francisco, CA, vol. 1, pp. 156163, March 1996.

[11] E. Karasan and E. Ayanoglu, "Effects of Wavelength Routing and Selection Algorithms on Wavelength Conversion Gain in WDM Optical Networks," IEEE/ACM Transactions on Networking, vol. 6, no. 2, pp. 186196, April 1998.

[12] S. Subramaniam and R. A. Barry, "Wavelength Assignment in Fixed Routing WDM Networks, Proc., ICC '97, Montreal, Canada, vol. 1, pp. 406410, June 1997.

[13] X. Zhang and C. Qiao, "Wavelength Assignment for Dynamic Traffic in Multi-fiber WDM Networks,"Proc., 7th International Conference on Computer Communications and Networks, Lafayette, LA, pp. 479- 485, Oct. 1998.

[14] Katz, D., Kompella, K., Yeung, D., Traffic Engineering (TE) Extensions to OSPF Version 2 RFC 3630, Setembro de 2003.

[15] Lang, J. E., Link Management Protocol RFC 4204, Outubro de 2005

[16] Mannie, E., Papadimitriou, D. Recovery (Protection and Restoration) Terminology for Generalized Multi-Protocol Label Switching (GMPLS) RFC 4427, Março de 2006.

[17] GMPLS Architecture and Applications - Adrian Farrel and Igor Bryskin, pag 89.

[18] Vasseur, J.-P., Pickavet, M., Demeester, P. Network Recovery: Protection and Restoration of Optical, SONET-SDH and MPLS. Morgan Kaufmann, 2004.

[19] J.C.R.F. Oliveira, J.R.F Oliveira, L.R. Monte, G.C. Santos e R. Arradi, "Esquema de Proteção Óptica Rápida para Redes Reconfiguráveis Baseadas em ROADMs de Grau 2", MOMAG 2008, Florianópolis, Brasil.

[20] Braden, E. R., Zhang, L., Berson, S., Herzog, S., Jamin, S., Resource ReSerVation Protocol (RSVP) RFC2205, Setembro de 1997. 